\title{
Numerical Analysis of Rotating Pumping Flows in Inter-Coil Rotor Cavities and Short Cooling Grooves of a Generator
}

\author{
WEI TONG* \\ Power Systems Division, General Electric Company, Schenectady, New York 12345, USA
}

(Received 8 April 1999; In final form 16 September 1999)

\begin{abstract}
An important characteristic of wall rotating-driven flows is the tendency of fluid with high angular momentum to be flung radially outward. For a generator, the rotor rotating-driven flow, usually referred to as the rotating pumping flow, plays an important role in rotor winding cooling. In this study, three-dimensional numerical analyses are presented for turbulent pumping flow in the inter-coil rotor cavity and short cooling grooves of a generator. Calculations of the flow field and the mass flux distribution through the grooves were carried out in a sequence of four related cases under an isothermal condition: (a) pumping flow, which is the self-generated flow resulted from the rotor pumping action; (b) mixing flow, which is the combination of the ventilating flow and pumping flow, under a constant density condition; (c) mixing flow, with density modeled by the ideal gas law; and (d) mixing flow, with different pressure differentials applied on the system. The comparisons of the results from these cases can provide useful information regarding the impacts of the ventilating flow, gas density, and system pressure differential on the mass flux distribution in the short cooling grooves. Results show that the pumping effect is strong enough to generate the cooling flow for rotor winding cooling. Therefore, for small- or mid-size generators ventilation fans may be eliminated. It also suggests that increasing the chimney dimension can improve the distribution uniformity of mass flux through the cooling grooves.
\end{abstract}

Keywords: Rotating pumping flow, Ventilating flow, Mixing flow, Inter-coil rotor cavity, Short cooling groove, Mass flux distribution

\section{INTRODUCTION}

The increasing demands for power supply have accelerated the design of reliable, high-powered, high-efficiency, and cost-effective modern generators with state-of-the-art technologies. Among many design parameters, thermal capability and insulation are recognized as two major items to limit the generators' rating and size. In order to ensure reliable operations of a generator,

\footnotetext{
* E-mail: tongwe $(a)$ pssch.ps.ge.com.
} 
the cooling/ventilation system in the generator plays a crucial role to maintain the generator temperatures within specified limits.

There are two kinds of cooling methods used in generator field winding cooling: indirect (or conventional) cooling and direct cooling. Indirect cooling was the first method developed to cool the field winding. It relies on heat conduction from the copper conductors through the insulation to the relatively cooler rotor core. Heat is then removed by cooling gas flowing through the vent slots in the rotor teeth. Direct cooling is implemented in two different ways: (a) Radial flow cooling uses the rotor rotation to create a pressure differential between the subslot and the stator-rotor air gap, and thus to force gas radially outward through rotor slots into the rotor winding. (b) Diagonal flow cooling uses the rotor surface velocity to force gas through the rotor winding turns. Cooling gas is scooped out of the stator-rotor gap by inclined holes in the coil wedges and forced to flow radially inward and along the axis of the rotor. At the bottom of the slot, air flows diagonally outward until it exhausts into the air gap once again (Nold and Fogarty, 1991; Tornroos and Randall, 1992).

As a more effective and efficient cooling method, direct cooling is widely used in rotor windings to attain a relative uniform temperature along the conductor length. Radial flow cooling can be achieved by machining ventilating grooves on the copper conductors. As the conductors are wound into a coil and further assembled into a rotor winding, these grooves provide paths for cooling fluids to cool the rotor winding.

Rotating flows are commonly encountered in rotary machines such as turbomachines, pumps, generators, and a variety of other engineering applications. A firm understanding of such rotating pumping flows leads directly to a fundamental understanding of the nature of rotary energy systems and their operations. However, in many circumstances, because of the problem complexity, technique restriction, and resource limitation (such as time, expenses, and test equipment), an experimental test becomes very difficult and thus a numerical investigation is a feasible choice for providing insight into the flow field. Over the past decade, the use of CFD tools has increased significantly by generator engineers and designers, resulting in a short design cycle time and low cost for the new generator introduction. The discipline of CFD has fostered a unified approach to turbomachinery analysis and design. The comprehensive reviews of the computation and modeling of turbomachinery flows were provided by Childs (1993) and Lakshminarayana (1996).

This work is a continuous effort for optimizing the generator design and for enhancing the generator cooling efficiency. The study was structured and performed as a sequence of four related cases: (a) Pumping flow, which is a self-generated flow by the pumping action of the rotor. To eliminate the influence of the ventilating flow in the system, the pressure differential between the flow inlet and the exit was set at zero. (b) Mixing flow, which is a combination of the pumping and ventilating flows in dual cooling. In this case, constant density is assumed. (c) As same as (b) but modeling density with the ideal gas law. The comparison between (b) and (c) can determine the effect of gas density on the mass flux and its distribution through grooves. (d) As same as (c) but varying $p_{\mathrm{i}} \pm 20 \%$ to its base value while keeping $p_{\mathrm{e}}$ constant. In such a way, the sensitivity of mass flux to the variation of the pressure differential can be identified.

It is worthwhile to note that as a preliminary study, this work is limited to isothermal flows under standard conditions $\left(p_{\mathrm{op}}=1 \mathrm{~atm}\right.$ and $T=$ $288.16 \mathrm{~K}$ ). In a real case, because of the impacts of heat transfer and gas compressibility, the flow and pressure fields might be different from these of the current analysis.

\section{MATHEMATICAL MODEL}

The schematic diagram of a rotor winding assembly and short cooling grooves is shown in Fig. 1. The rotor winding consists of a number of coils, separated by wedge blocks along the rotor axis and 


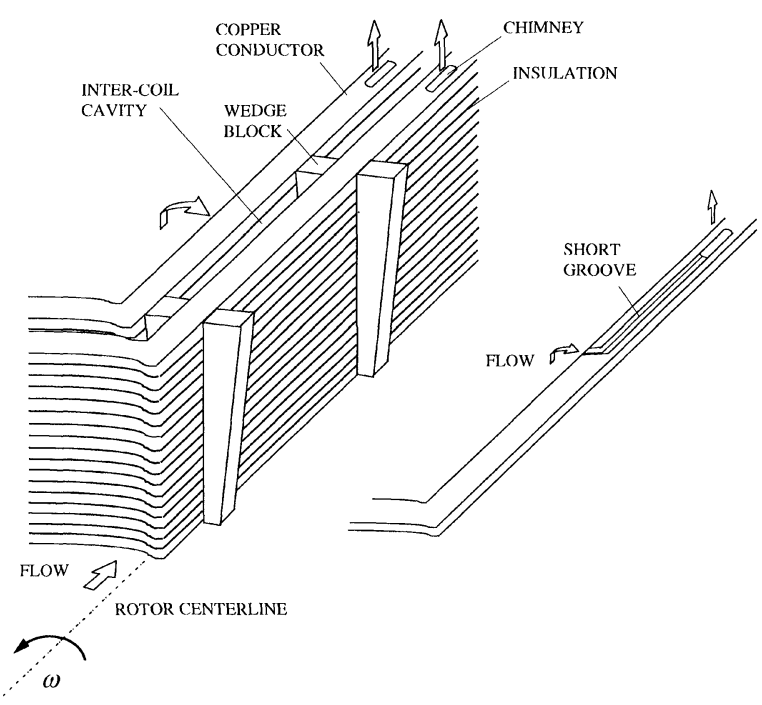

FIGURE 1 Schematic diagram of the rotor winding assembly and short cooling grooves.

space blocks at the front of the end-winding. Hence, a number of rotor cavities are formed between adjacent coils and wedge blocks (or space blocks). A coil is wound by copper conductors with insulation between adjacent turns. There are seven short grooves in each coil, arranged at the odd turns from \#3 to \#15 (counted from top to bottom). A short groove starts at the wedge block with $45^{\circ}$ to the conductor axial centerline, then becomes parallel to the conductor, and eventually terminates at the radial cooling "chimney." This cooling arrangement brings the cold gas into direct contact with conductors and eliminates the thermal resistance through the insulation. A cooling gas from the environment enters into the subslot cavity, which is beneath the end-windings, fills the inter-coil rotor cavity, distributes into short grooves, then discharges into the chimney, and exhausts to the rotor-stator air gap. Since all coils are ventilated in the similar manner, only one coil is modeled in this work. Air is used as the cooling fluid.

In the present work, the Renormalization Group (RNG) $k-\varepsilon$ turbulent model was used because it can provide greater accuracy in modeling rotating and swirl flows. Unlike the standard $k-\varepsilon$ model that is based on Reynolds averaging, this model is derived from the instantaneous Navier-Stokes equations. The basic idea of this method is the elimination of small-scale eddies. It can provide improved predictions of turbulent flow in the gap. The solution-adaptive mesh refinement strategy was also used to add cells in the vicinity of the rotor surface. Implementation of this method was observed to be very effective in increasing the numerical calculation accuracy without requiring unnecessary computational resources (FLUENT, 1997a).

The governing equations for a three-dimensional, steady-state, isothermal, turbulent flow are

$$
\nabla \cdot(\rho \mathbf{u})=0
$$

$$
\begin{aligned}
\mathbf{u} \cdot \nabla(\rho \mathbf{u})= & -\nabla p+2 \rho \mathbf{u} \times \mathbf{\Omega}+\nabla\left[\frac{\mathbf{1}}{\mathbf{2}} \rho(\mathbf{\Omega} \times \mathbf{r})^{2}\right] \\
& +\nabla\left[\mu_{\mathrm{eff}}\left(\nabla \mathbf{u}+\nabla \mathbf{u}^{\mathrm{T}}\right)\right] \\
\nabla(\rho \mathbf{u} k)= & \nabla\left(\alpha_{k} \mu_{\mathrm{eff}} \nabla k\right)+\mu_{\mathrm{t}} \Phi-\rho \varepsilon \\
\nabla(\rho \mathbf{u} \varepsilon)= & \nabla\left(\alpha_{\varepsilon} \mu_{\mathrm{eff}} \nabla \varepsilon\right)+c_{1} \frac{\varepsilon}{k} \mu_{\mathrm{t}} \Phi \\
& -c_{2} \rho \frac{\varepsilon^{2}}{k}-R
\end{aligned}
$$

where $\varepsilon$ and $k$ are known as the turbulent kinetic energy and the dissipation rate of the turbulent kinetic energy, $\mu_{\mathrm{t}} \Phi$ is the turbulent viscous dissipation, $\boldsymbol{\Omega}=-\omega \mathbf{k}$ is the angular velocity vector, $\alpha_{k}$ and $\alpha_{\varepsilon}$ denote the inverse Prandtl number for $k$ and $\varepsilon$, respectively, $\mu_{\mathrm{t}}$ and $\mu_{\mathrm{eff}}$ are the turbulent and effective viscosity (Launder and Spalding, 1974), respectively,

$$
\begin{aligned}
\mu_{\mathrm{t}} & =\rho C_{\mu} \frac{k^{2}}{\varepsilon} \\
\mu_{\mathrm{eff}} & =\mu_{\mathrm{mol}}\left[1+\sqrt{\frac{\rho C_{\mu}}{\mu_{\mathrm{mol}}}} \frac{k}{\sqrt{\varepsilon}}\right]^{2}
\end{aligned}
$$

The terms $2 \rho \mathbf{u} \times \boldsymbol{\Omega}$ and $\nabla\left[1 / 2 \rho(\boldsymbol{\Omega} \times \mathbf{r})^{2}\right]$ in Eq. (2) are the Coriolis and centrifugal forces per unit volume of the fluid, respectively (Landau and Lifshits, 1998). It is easy to prove that the centrifugal force 
TABLE I Constants in the RNG $k-\varepsilon$ Model

\begin{tabular}{cccc}
\hline$C_{1}$ & $C_{2}$ & $\alpha_{k}$ & $\alpha_{\varepsilon}$ \\
\hline 1.42 & 1.68 & 1.393 & 1.393 \\
\hline
\end{tabular}

can be expressed as the product of density $\rho$ and centripetal acceleration $\boldsymbol{\Omega} \times(\boldsymbol{\Omega} \times \mathbf{r})$, i.e.,

$$
\nabla\left[\frac{1}{2} \rho(\boldsymbol{\Omega} \times \mathbf{r})^{2}\right]=-\rho \boldsymbol{\Omega} \times(\boldsymbol{\Omega} \times \mathbf{r})
$$

The last term in Eq. (4) is the rate-of-strain, defined as

$$
R=\frac{C_{\mu} \eta^{3}\left(1-\eta / \eta_{0}\right)}{1+\zeta \eta^{3}} \frac{\varepsilon^{2}}{k}
$$

where, $\eta \equiv S k / \varepsilon, \eta_{0} \approx 4.38, \zeta=0.012$.

The constants contained in the governing equations are given in Table I.

It is noted that when the RNG model is used, the effect of rotation on turbulence must be accounted by modifying $C_{\mu}$ as a function of angular velocity $\omega$, $k / \varepsilon$ ratio, and swirl constant $\alpha_{\mathrm{s}}$,

$$
C_{\mu}=C_{\mu_{0}} f\left(\alpha_{s}, \omega, k / \varepsilon\right)
$$

where $C_{\mu_{0}}=0.0845$ and $\alpha_{\mathrm{s}}$ assumes different values depending on whether the flow is swirl-dominated or only mildly swirling (FLUENT, 1997a).

The angular rotating speed with respect to the rotor axis was set at 314 radians per second $(\mathrm{rpm}=3000)$ for the whole computational domain. Periodic rotating boundary conditions were applied in the circumferential direction on the periodic walls of the subslot cavity beneath the end-winding. The pressure differential between the flow inlet and the exit was set differently, varied from case to case.

\section{NUMERICAL METHODS}

Equations (1)-(4) were formulated via a finite volume CFD code known as FLUENT (1997b). This code offers a wide of choice of modeling technologies for turbomachines and has been well tested in designs and analyses in engineering applications.

The total computational domain was effectively meshed by dividing it into four parts: subslot cavity, inter-coil cavity, short grooves, and radial chimney. Grid sizes were carefully selected for each part to adequately resolve the internal details. Very fine meshes were made at the short grooves and the chimney, as well as near-wall regions (Fig. 2). More than 135,000 hexahedral meshes were employed in this work. All computations were performed on an HP-C160 workstation.

A two-layer-based non-equilibrium wall function was used to resolve flows in the near-wall region. This approach employs the two-layer concept in computing the budget of turbulent kinetic energy at the wall-adjacent cells. The wall-neighboring cells consist of a viscous sublayer and a fully turbulent layer. By applying the non-equilibrium wall function, it is important to maintain $y^{+}$(where $y^{+}=$ $\left.\delta c_{\mu}^{1 / 4} k^{1 / 2} / \nu\right)$ in the appropriate range over which the log-law is valid.

The main difficulties encountered in simulating such a complex, three-dimensional, turbulent flow were the solution stability and convergence. Several computational techniques were used to accelerate the convergence rate and improve solution stability. The most effective way to control solution stability was to start the rotating speed at a very low level $(15-20 \%$ of its final value) and increase it slowly in subsequent runs. This procedure continued until that the desired rotating speed was reached. Moreover, the underrelaxation parameters were set at $0.2-0.3$ for continuity, momentum, $k$ and $\varepsilon$. A calculation terminates when all maximum residues of continuity, momentum, $k$ and $\varepsilon$ are less than $5 \times 10^{-4}$.

\section{RESULTS AND DISCUSSION}

\section{Pumping Flow}

When a rotor is rotating at a constant angular speed, a pressure gradient, as well as a velocity 


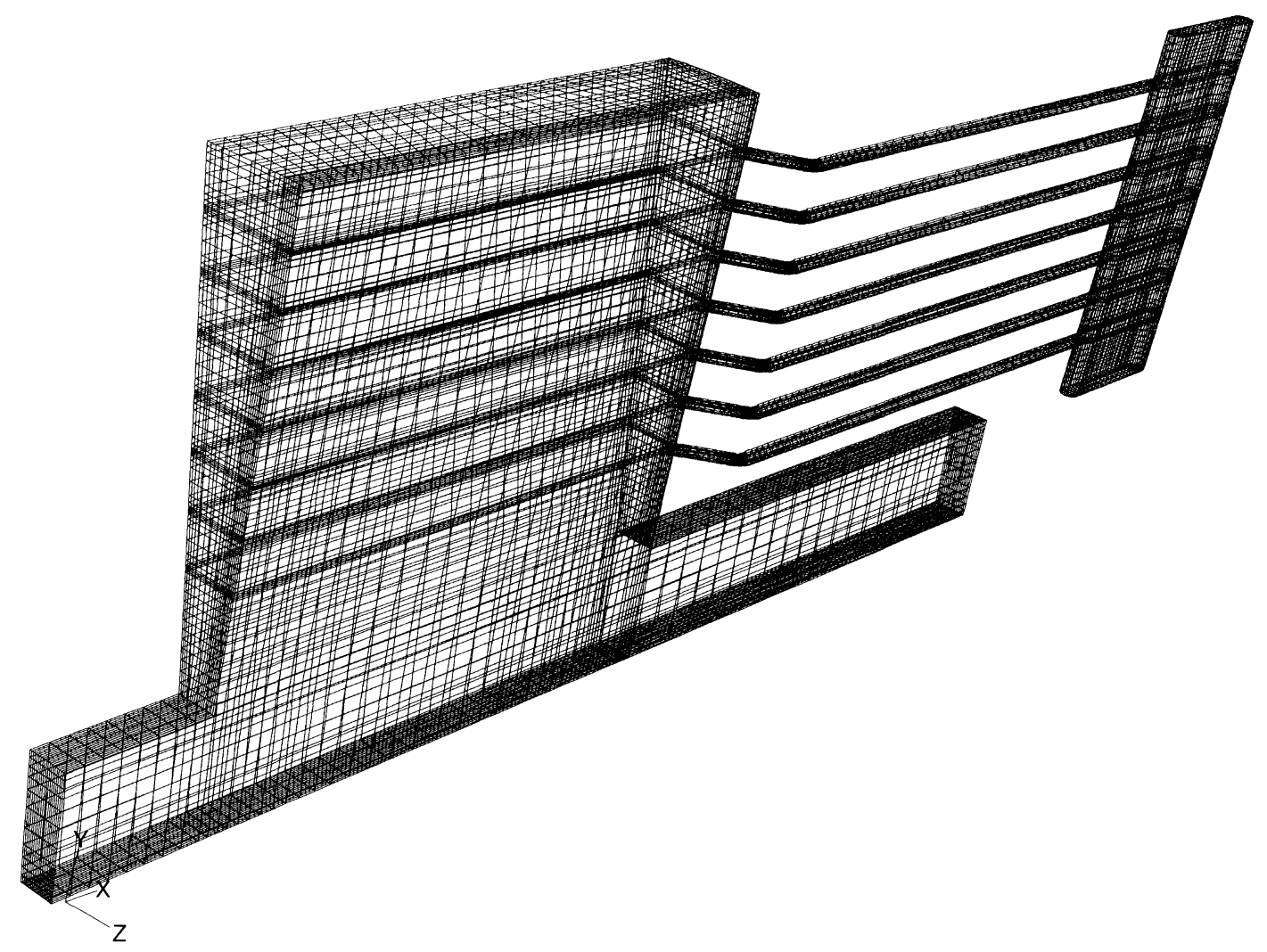

FIGURE 2 Computational domain and wall mesh setting.

gradient, is set up in the radial direction. Once a flow inlet and an exit are provided, this pressure gradient will drive cold air to flow through the system and pump it out of the exit. In order to limit the proposed solutions only to the pumping flow, the pressure differential between the flow inlet and the chimney exit was set at zero. Thus, the flow is merely driven and accelerated by the rotating pumping action. In this case, constant air density $\left(\rho=1.225 \mathrm{~kg} / \mathrm{m}^{3}\right)$ was used for all calculations.

A total pressure is defined as the sum of static pressure $p_{\mathrm{s}}$ and dynamic pressure $p_{\mathrm{d}}\left(p_{\mathrm{d}}=\rho|\mathbf{u}|^{2} / 2\right)$. The distribution of the static pressure (which is relative to the operating pressure) of the pumping flow is shown in Fig. 3(a). It can be observed that the static pressure distributes almost linearly with the radius in the inter-coil cavity, subslot cavity, and along the chimney. The maximum static pressure occurs at the top of the inter-coil rotor cavity and the minimum occurs at the dead region at the chimney bottom. Inside a groove, the static pressure gradient is established linearly along the groove length. Figure 3(b) displays the dynamic pressure distribution in the system. Because $p_{\mathrm{d}}$ is directly associated with the fluid velocity (primarily tangential velocity), the dynamic pressure is approximately proportional to the radius in the inter-coil cavity. However, in the subslot cavity, due to the impact of the inlet velocity the dynamic pressure field is completely distorted. Similarly, because of the interactions among the air jets, as well as those between the jets and the chimney wall, both the static and dynamic pressure fields in the chimney are distorted.

Velocity vectors in the chimney and short grooves are displayed in Fig. 4. When air ejects from a groove into the chimney, it maintains the mean stream for a certain distance, and then 

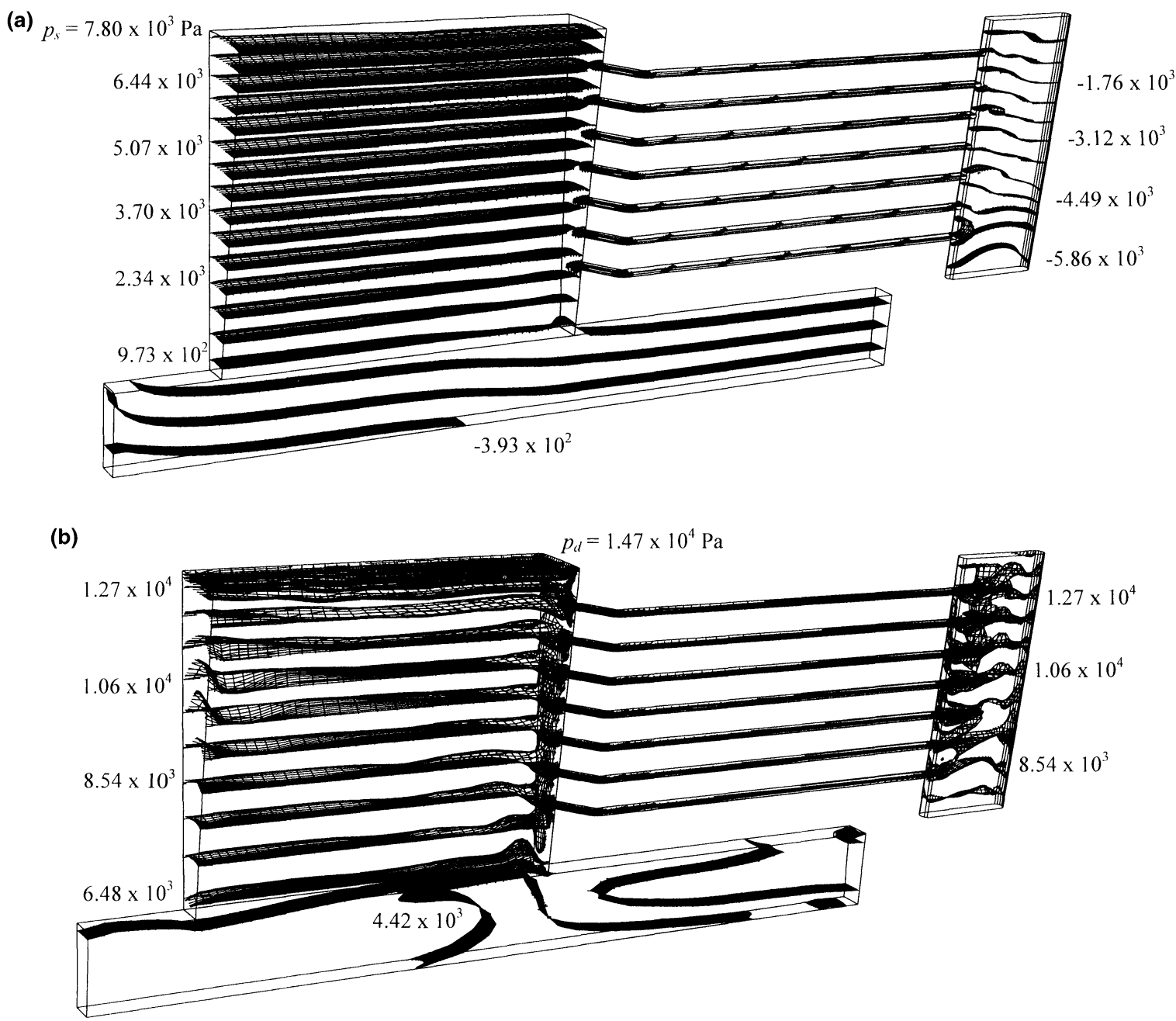

FIGURE 3 (a) Static pressure distribution of the pumping flow. (b) Dynamic pressure distribution of the pumping flow.

interacts with the chimney wall to change the flow direction towards the exit. A flow circulation is formed in the dead region at the chimney bottom.

The flow pattern through a groove can be characterized by Reynolds number, which is defined based on the groove hydraulic diameter $D_{\mathrm{h}}$, mass flux $G$, and effective dynamic viscosity $\mu_{\text {eff }}$,

$$
R e=\frac{G D_{\mathrm{h}}}{\mu_{\mathrm{eff}}}
$$

For the pumping flow, $R e$ is about $10^{4}$ in the cooling grooves.
One of the major parameters for determining the mass flowrate through a groove is the pressure differential across the groove. Numerical results show that since the pressure is approximately a linear function of the radius in the inter-coil rotor cavity, it is the distorted pressure field in the chimney that causes the difference in $\Delta p$ among the different grooves, and in turn, the different mass flowrate through these grooves.

All cooling grooves are designed identical in both size and shape to ensure the flow uniformity. However, deviations may be introduced at the geometry modeling and mesh setting. To eliminate the impact 


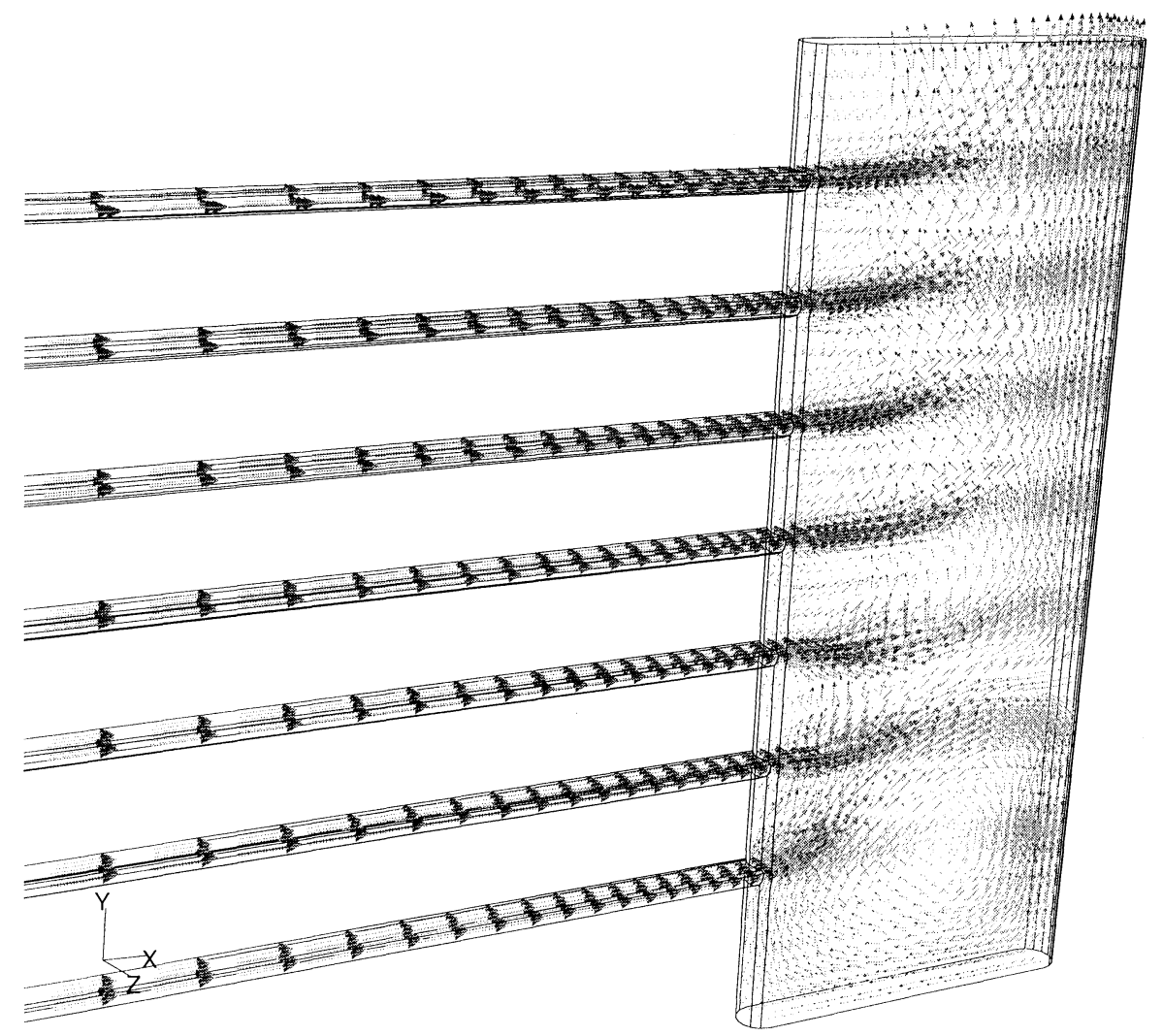

FIGURE 4 Velocity vectors through the short cooling grooves and chimney for the pumping flow.

of such deviations on mass flowrate $\dot{m}$, mass flux $G$, which is defined as mass flowrate per unit area, was used in this work to characterize the flowrate distribution,

$$
G=\frac{\dot{m}}{A}
$$

where mass flowrate $\dot{m}$ is computed by integrating the product of density with the dot product of the facet area vector and the facet velocity vector, i.e.,

$$
\dot{m}=\int \rho \mathbf{u} \cdot \mathrm{d} \mathbf{A}
$$

The variation of the mass flux through short grooves is shown in Fig. 5. The mean mass flux is $65.48 \mathrm{~kg} /\left(\mathrm{m}^{2} \mathrm{~s}\right)$ with the standard deviation of 0.736 . This may suggest that for small and medium

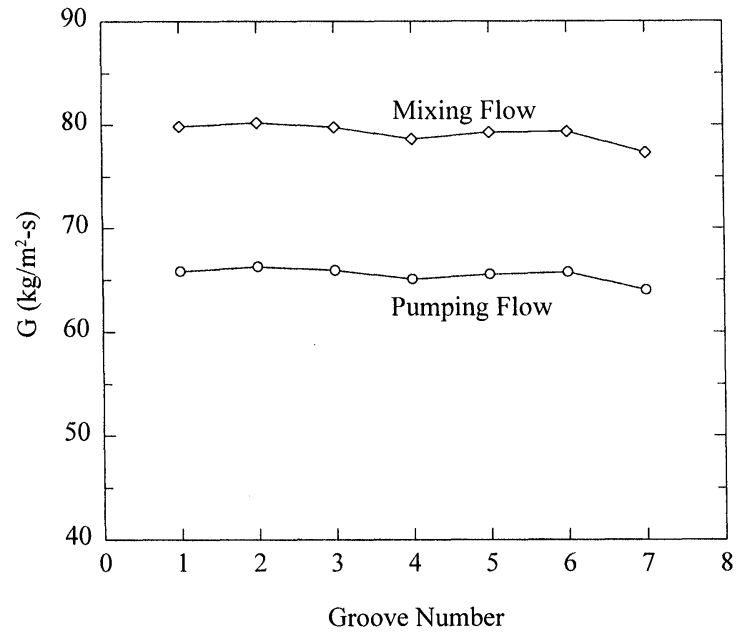

FIGURE 5 Comparison of the mass flux and its distribution through short cooling grooves between pumping and mixing flows. 
generators the pumping flow is sufficient for the rotor winding cooling and thus, rotor fans may be possibly eliminated. The mass flux distribution is rather uniform, with all data falling into a $\pm 2.2 \%$ band regarding its mean value. A lowest mass flux value occurs at the groove \#7 at the low pressure region.

\section{Mixing Flow}

When pressures at the flow inlet and exit are set differently, the ventilating flow arises in the system and superimposes with the pumping flow as a mixing flow. By maintaining all other boundary conditions identical as in the previous case, the applied pressure differential will lead to the increase in mass flux, and consequently, enhance the cooling performance for the rotor windings.

It can be seen from Fig. 5 that as $\Delta p=127.5 \mathrm{~Pa}$ $\left(p_{\mathrm{i}}=166.6\right.$ and $\left.p_{\mathrm{e}}=39.1 \mathrm{~Pa}\right)$, the mass flux increases approximately $20 \%$ (where $G=79.21 \mathrm{~kg}$ / $\left.\left(\mathrm{m}^{2} \mathrm{~s}\right)\right)$ to the pumping flow. However, it shows clearly that the superposition of the ventilation flow has little influence on the mass flux distribution.

Similarly, the calculated results show that the superposition of the ventilating flow to the pumping flow increases significantly the pressure magnitude but has little impact on the pressure distribution pattern. With $\Delta p$ applied on the system, the maximum and minimum pressure have increased approximately $58 \%$ and $20 \%$ respectively to those in the pumping flow. By comparing the velocity vectors for the pumping flow, the increase in mass flux causes much stronger interactions between jets and the chimney wall.

\section{Effect of Gas Density on Mass Flux Distribution}

In an attempt to examine the gas density variation on the mass flux and its distribution, equation of state for a single-component ideal gas is used to calculate the gas density,

$$
\rho=\frac{\left(p_{\mathrm{op}}+p_{\mathrm{s}}\right) M}{R_{\mathrm{u}} T}
$$

where $M$ is the molecular weight of air, $R_{\mathrm{u}}$ is the universal gas constant, $p_{\mathrm{s}}$ is the local static pressure relative to the operating pressure $p_{\text {op }}$. Thus, for isothermal flows density becomes a linear function of pressure. As a result, the distribution pattern of density should be identical to that of pressure. From Fig. 6, it can be seen that iso-density plans run parallel to the rotor surface in the inter-coil rotor cavity. With a high density region (where $\rho>1.225 \mathrm{~kg} / \mathrm{m}^{3}$ ) occurring at the inter-coil rotor cavity and a low density region (where $\rho<1.225 \mathrm{~kg}$ / $\mathrm{m}^{3}$ ) in the chimney, an approximate linear density gradient is set up along the groove length. Following Eq. (11), the difference of mass flux between the constant and varied density cases can be expressed as

$$
\Delta G=(\rho u)_{\rho=c}-(\rho u)_{\rho \neq c}
$$

Numerical results have revealed that the relative change in velocity, $\Delta u / u$, between the two cases is approximately a constant $(1.7-1.9 \%)$ for all grooves and, in contrast, the relative change in density $\Delta \rho / \rho$ depends strongly upon the local pressure, ranging from $3 \%$ at the outermost groove (\#1) at the high pressure region to $5.5 \%$ at the innermost groove (\#7) at the low pressure region. Thus, the density variation becomes the key parameter to alter the mass flux and its distribution through grooves. Figure 7 shows the comparison of the mass flux, measured at the interfaces between the groove exits and the chimney wall, between the two cases. At these locations density in the varied density case is significantly lower than that in the constant density case, resulting in the decrease of the mass flux in the varied density case. From Fig. 7, $\Delta G$ is seen to vary with the groove location. As evidence, calculated results show that $\Delta G$ / $G=1.1 \%$ for the groove $\# 1$ and $5.1 \%$ for the groove \#7.

\section{Sensitivity of Applied Pressure Differential on Mass Flux and its Distribution}

The sensitivity of mass flux on pressure differential $\Delta p$ was studied by varying $p_{\mathrm{i}} \pm 20 \%$ to its base 


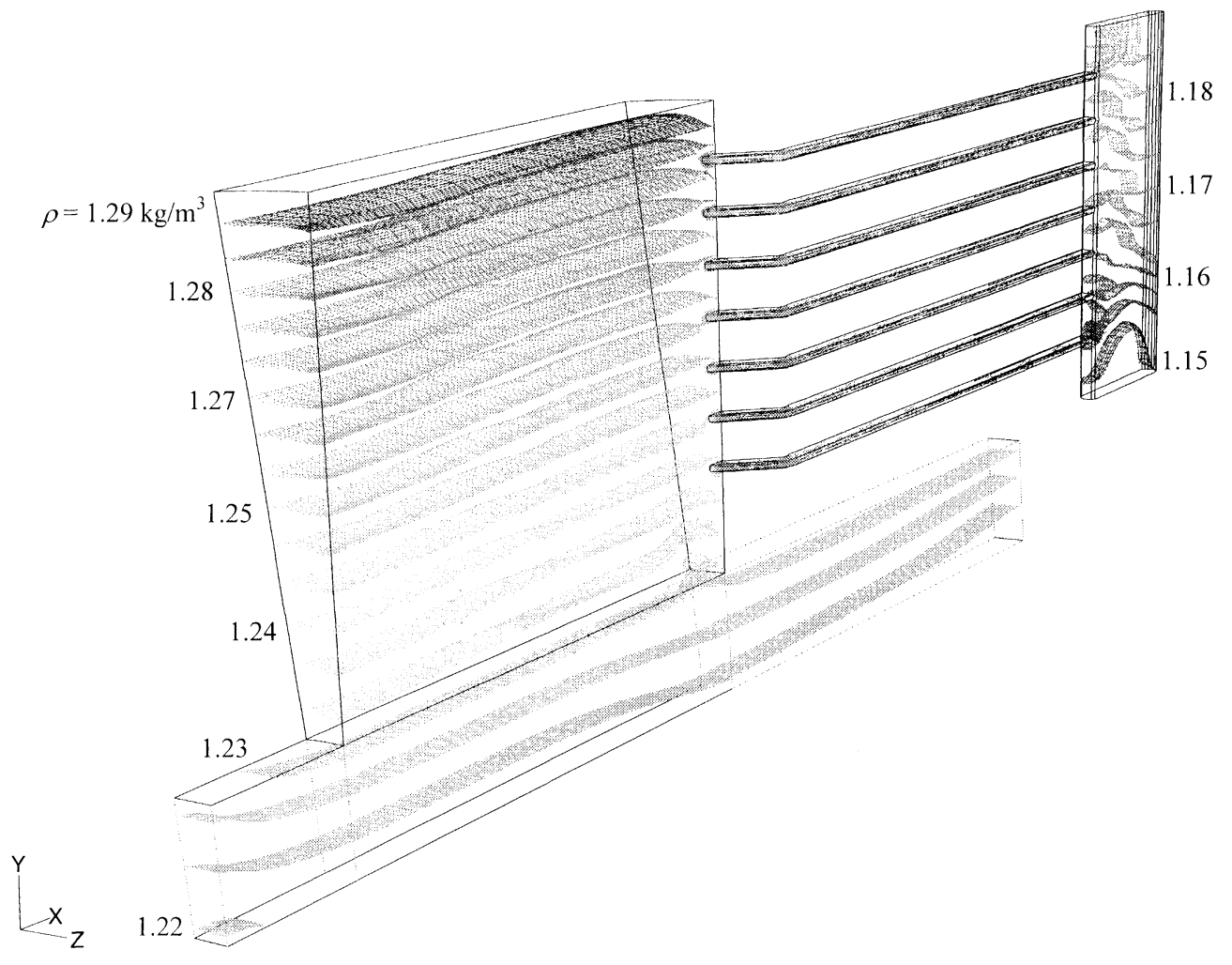

FIGURE 6 Density distribution of the mixing flow using the ideal gas law.

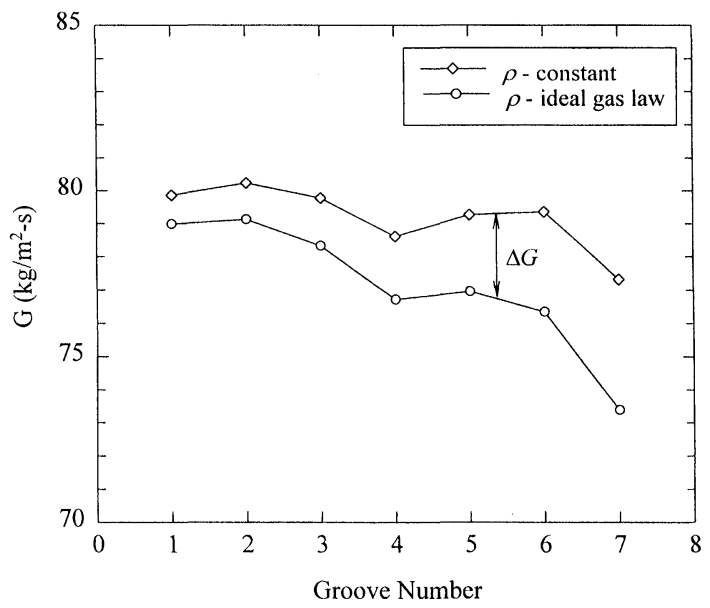

FIGURE 7 Effect of gas density variation on the mass flux and its distribution through short cooling grooves.

value while keeping $p_{\mathrm{e}}$ constant. In this case, density was modeled by the ideal gas law. Numerical results show that pressure differential can strongly affect mass flux in short grooves. When $p_{\mathrm{i}}$ changes $\pm 20 \%$, the mean mass flux changes correspondingly about $\pm 4.5 \%$. Thus, the sensitivity of mass flux to the variation of inlet pressure can be calculated as

$$
\frac{\Delta G / G}{\Delta p_{\mathrm{i}} / p_{\mathrm{i}}}=\frac{ \pm 4.5 \%}{ \pm 20 \%}=22.5 \%
$$

However, the profile of the mass flux distribution is found to be nearly independent of the pressure differential (Fig. 8).

\section{PRACTICAL IMPORTANCE}

The fan windage loss is the biggest part among all generator windage losses. The fan loss could count $62.6 \%$ of the total windage losses for an air-cooled generator and $63.8 \%$ for a hydrogen-cooled generator (Tong, 1998). In the design of advanced 


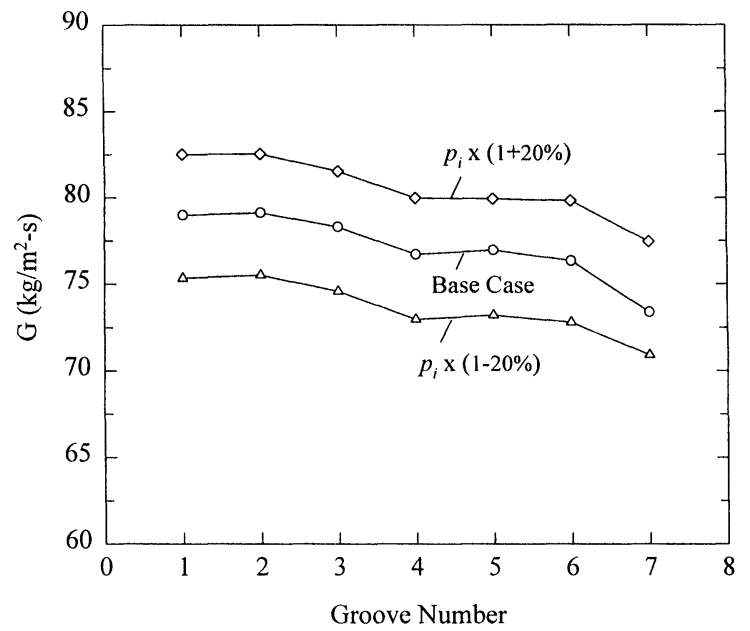

FIGURE 8 Effect of system pressure differential on the mass flux and its distribution through short cooling grooves.

modern generators, such fan windage losses should be desirably minimized or eliminated to enhance the generator performance efficiency.

The numerical results obtained from the present investigation can be used to evaluate the effect of the rotating pumping flow on the generator cooling. It has shown that as a pressure differential is applied on the system, the generated ventilating flow counts only a small portion to the total mixing flow. It appears that for small- or mid-size generators rotating pumping flows alone could meet the requirement for rotor winding cooling and thus, ventilation rotor fans may be eliminated.

In addition, the data of the mass flux distribution through the grooves suggest that increasing the chimney dimension can reduce the interactions between the air jets and chimney wall and thus, improve the distribution uniformity of mass flux through the grooves.

\section{CONCLUSIONS}

A numerical investigation of the turbulent flow and pressure fields in the inter-coil rotor cavity and short cooling grooves was performed under an isothermal condition. With a three-dimensional CFD model, the flow pattern in the system and the mass flux distribution though cooling grooves were obtained. The major conclusions from the numerical results can be drawn as follows:

(1) The pumping effect was demonstrated to be considerably strong enough to generate air cooling flows in a generator. It shows even a large pressure differential applied on the system, the pumping flow still counts about $4 / 5$ to the total cooling flow.

(2) When a ventilating flow is introduced by applying a pressure differential on the system, increasing the pressure differential can result in an increase in mass flux. However, the superposition of the ventilating flow has shown little influence on the mass flux distribution though the grooves.

(3) The variation of the gas density can fairly alter both the mass flux magnitude and its distribution. By using the ideal gas law, density becomes a linear function of pressure for isothermal flows. The change in mass flux is mainly caused by the density variation.

(4) Sensitivity of the pressure differential on the mass flux distribution was examined by varying $p_{\mathrm{i}} \pm 20 \%$ to its base value while keeping $p_{\mathrm{e}}$ at constant. Sensitivity of mass flux to the variation of the air inlet pressure was $22.5 \%$. In addition, the distribution pattern of mass flux was found almost independent of the pressure drop applied on the system.

(5) The numerical results have shown that the total pressure distributes approximately linearly in the inter-coil rotor cavity. It is the distorted pressure field in the chimney that causes the difference in mass flux through cooling grooves.

\section{NOMENCLATURE}
A facet area vector
$A \quad$ flow area, $\mathrm{m}^{2}$
$C_{1}, C_{2}, C_{\mu} \quad$ turbulent model constants
$D_{\mathrm{h}} \quad$ hydraulic diameter, $\mathrm{m}$ 
G

$k$

$L$

$M$

$\dot{m}$

$p$

Re

$R_{\mathrm{u}}$

$\mathbf{r}$

$T$

$\mathbf{u}$

$y^{+}$ mass flux, $\mathrm{kg} /\left(\mathrm{m}^{2} \mathrm{~s}\right)$

turbulent kinetic energy, $\mathbf{J}$

length, $\mathrm{m}$

molecular weight, kgmol

mass flowrate, $\mathrm{kg} / \mathrm{s}$

pressure, $\mathrm{Pa}$

Reynolds number, $R e=G D_{\mathrm{h}} / \mu_{\text {eff }}$

universal gas constant,

$$
=8.314 \mathrm{~kJ} /(\mathrm{kgmol} \cdot \mathrm{K})
$$

position vector

temperature, $\mathrm{K}$

velocity vector

non-dimensional distance of nearwall cell center, $y^{+}=\delta C_{\mu}^{1 / 4} k^{1 / 2} / \nu$

\section{Greek Symbols}

$\alpha_{k}, \alpha_{\varepsilon} \quad$ turbulent model constants

$\alpha_{\mathrm{s}} \quad$ swirl constant

$\delta \quad$ distance of near-wall cell center from the wall, $m$

$\varepsilon \quad$ viscous dissipation rate of turbulent kinetic energy, W

$\rho \quad$ density, $\mathrm{kg} / \mathrm{m}^{3}$

$\mu \quad$ dynamic viscosity, $\mathrm{Pa}$ s

$\nu \quad$ kinematic viscosity, $\mathrm{m}^{2} / \mathrm{s}$

$\boldsymbol{\Omega} \quad$ angular velocity vector

$\omega \quad$ angular velocity, $\mathrm{rad} / \mathrm{s}$

\section{Subscripts}

d dynamic

e exit

eff effective

i inlet

mol molecular

op operating

s static

$\mathrm{t}$ turbulent

\section{References}

Childs, D. (1993) Turbomachinery Rotordynamics: Phenomena, Modeling, and Analysis, John Wiley, New York.

FLUENT (1997a) Physical models for flow and heat transfer in FLUENT/UNS, Chapter 8, User's Guide for FLUENT/UNS \& RAMPANT 4.2, Vol. 2.

FLUENT (1997b) Getting started, Chapter 1, User's Guide for FLUENT/UNS \& RAMPANT 4.2, Vol. 1.

Lakshminarayana, B. (1996) Fluid Dynamics and Heat Transfer of Turbomachinery, John Wiley, New York.

Landau, L.D. and Lifshits, E.M. (1998) Fluid Mechanics, 2nd edn., Pergamon, New York.

Launder, B.E. and Spalding, D.B. (1974) The numerical computation of turbulent flow, Comp. Meth. App. Mech. Eng. 3, 269-289.

Nold, R.A. and Fogarty, J.M. (1971) Introduction to basic concepts for generators, GE Power Systems Technical Report TGE 91-72.

Tong, W. (1998) Evaluating generator air gap profile effects on windage losses, GE Power Systems Technical Report TGE 98-20.

Tornroos, K.C. and Randall, J.H. (1992) Overall thermal design of medium and large generators, GE Power Systems Technical Report TGE 92-3. 

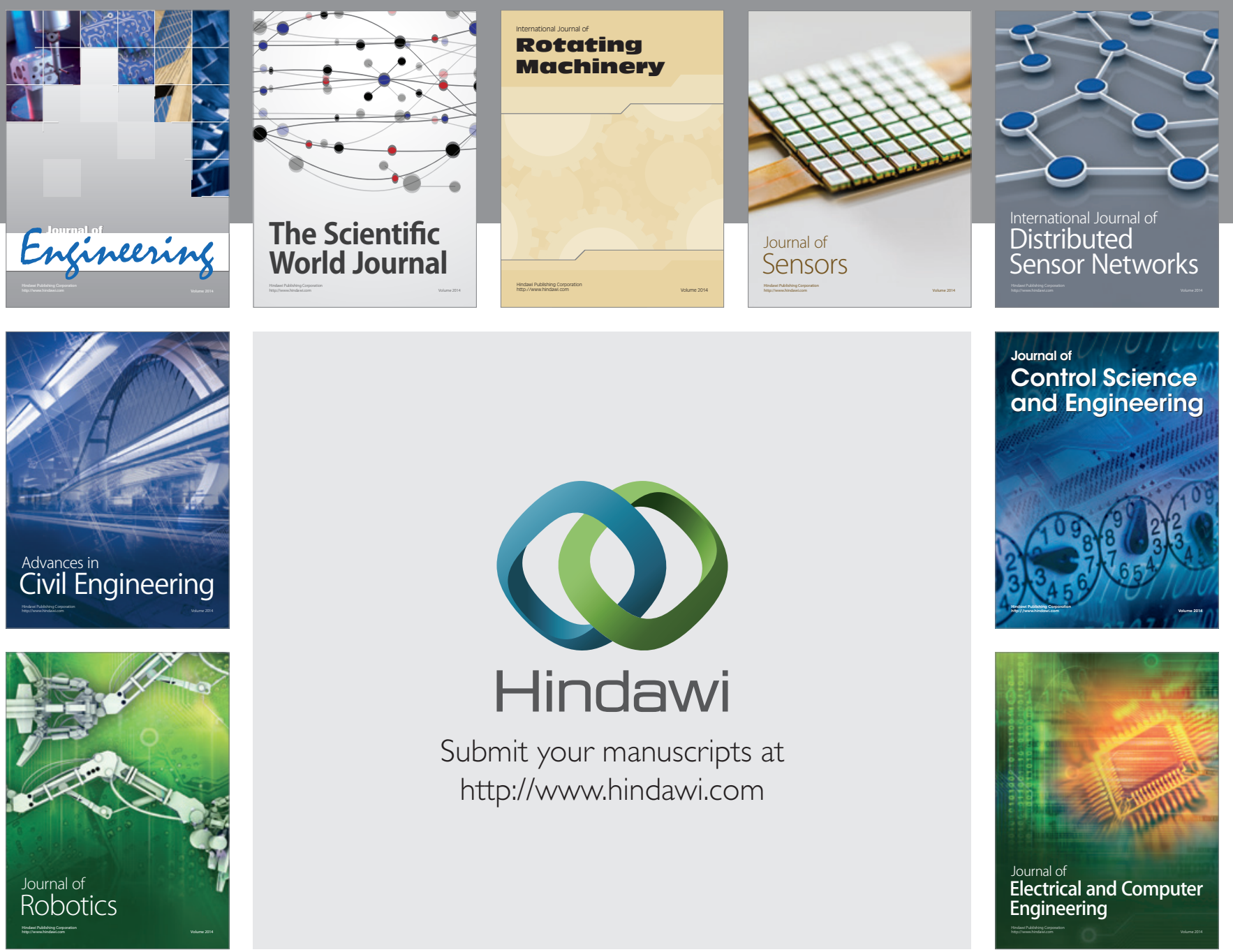

Submit your manuscripts at

http://www.hindawi.com
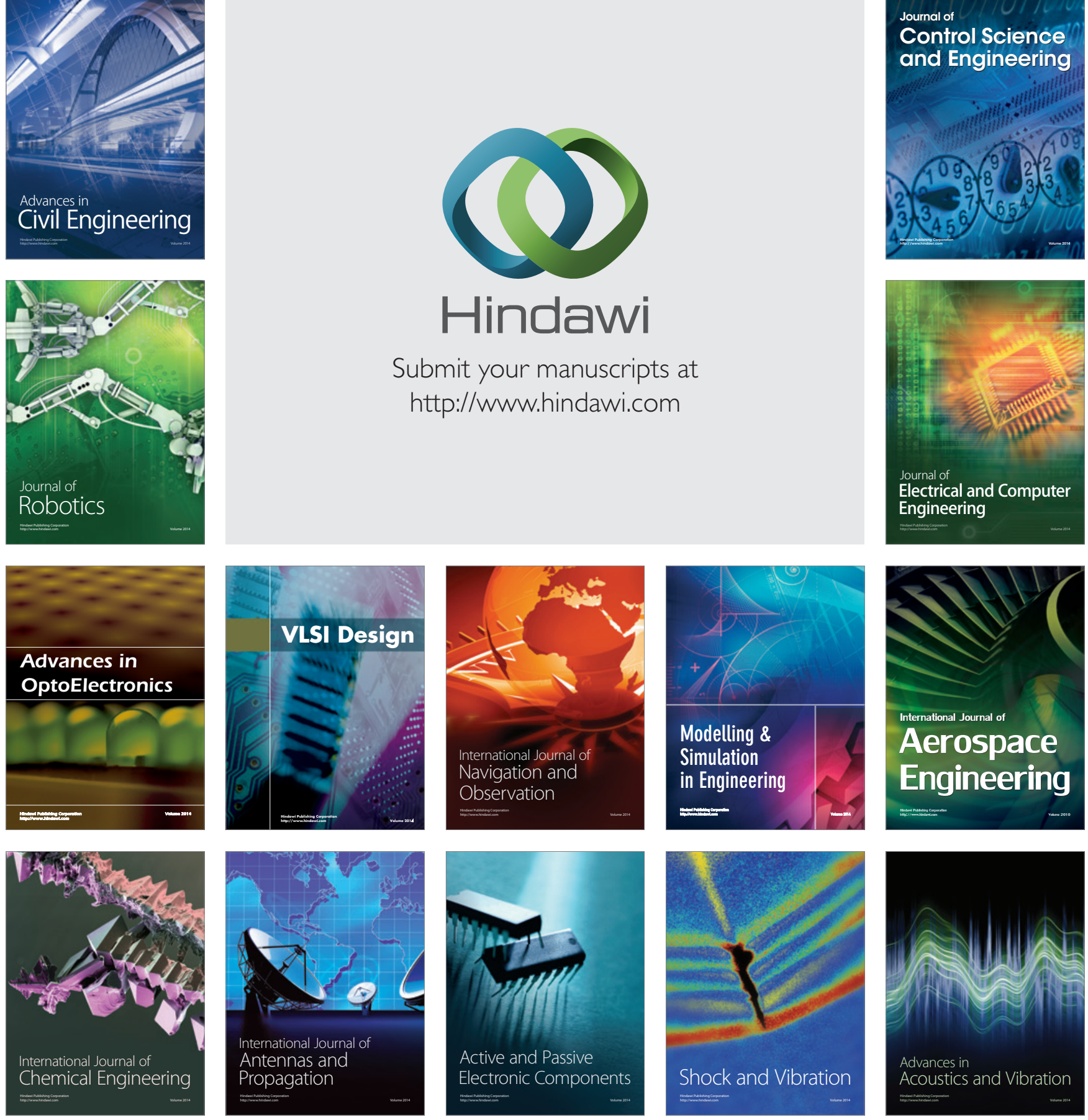